\title{
MODELING ON-SHORE SEDIMENT TRANSPORT USING ENERGETICS MODELS
}

\author{
Qun Zhao ${ }^{1}$, James T. Kirby ${ }^{1}$, Jack A Puleo ${ }^{1}$
}

\begin{abstract}
In this paper, a reformulation of the Bagnold sediment transport formula based on using friction velocity to express bottom shear stress is examined. This modification allows the transport formulation to retain the effect of phase lag between the free stream velocity and bottom shear stress usually associated with flow acceleration or pressure gradient, neglected in Bagnold's original formula. Unlike the original Bagnold model, the modified Bagnold model predicts net onshore sediment transport for asymmetric, zero skewness waves. Results for analytical waveforms of varying velocity amplitude and skewness are qualitatively similar to previous work based on discrete particle and two-phase flow methods. These comparisons suggest that if the simple energetics approach is to be employed, correct estimation of the bottom stress is paramount to predicting sediment transport with any accuracy.
\end{abstract}

Key words: BBB formula; on-shore sediment transport; free stream velocity

\section{INTRODUCTION}

Field observations (Gallagher et al. 1998, Elgar et al. 1998) suggest that nearshore sandbars migrate offshore under storm condition due to strong undertows caused by breaking waves over the bar, but migrate onshore between storms when unbroken waves are strongly skewed and undertow is weak. Models parameterized using free stream velocity statistics, such as the BBB formula (Bagnold, 1966; Bowen, 1980; Bailard, 1981), are often reported to be poor. Elgar et al. (2001) found that the onshore sediment transport is correlated to the skewness of the flow acceleration or velocity skewness. Hoefel and Elgar (2003) showed improved model skills when applying Drake and Calantoni(2001) semi-empirical term $\left\langle\mathbf{i}_{\text {spike }}\right\rangle$ to account for the flow acceleration.

The mechanisms on the cause of net onshore sediment transport under skewed waves are still not clear. Various theories and numerical models have been applied to study this problem. Nielsen and Callaghan (2003), Watanabe and Sato (2004), Nielsen (2006), etc. have focused on the phase lag effect on sheet flow, but the phase lag angle remains uncertain.

Numerical study of a two-phase flow model by Hsu and Hanes (2004) showed that although the magnitude of the pressure gradient may not be significant compared with the drag term, it remains important because the particle stress term effectively balances the drag term over the entire wave cycle. Calantoni and Puleo (2006) used a discrete particle model to more rigorously determine the effect of pressure gradients on sediment grains in the boundary layer. They found that the pressure gradient increases sediment transport in the onshore direction, though it is mainly the drag that is doing work on the grains. More important to onshore transport is the phasing of the peak in the pressure gradient that provides an additional push during offshore-onshore flow reversal under sawtooth waves. Using a 1-D eddy viscosity model, Henderson et al.(2004) showed that wave generated momentum fluxes and the Stokes drift substantially increased onshore sediment transport and were essential to predictions of onshore bar migration. More recently, Ruessink et al.(2009) used a 1D $k-\epsilon$ model to show that phase-lag effects are particularly important to fine sediment.

Gonzalez-Rodriguez and Madsen (2008) and Guard and Nielsen (2008) looked into the effect of a moveable bed on sediment transport and showed that conventional 'bed shear stress' is not well defined in unsteady flow. The appropriate bed roughness to parameterize the bed shear stress responsible for bedload sediment transport remains unclear.

Finally, Foster et al. (2006) suggested that when the Sleath parameter, which is the ratio of horizontal pressure gradient to gravity, exceeds the critical value of 0.29 , plug flow should be considered.

As shown above, the mechanisms causing onshore sediment transport under horizontal pressure gradient is still very much under research. All the mechanisms listed in the previous section may be reasonable under certain conditions or co-exist with each role. A correct parameterization considering all the mechanisms seems to be not attainable soon. In the present work, we aim at developing a simple bulk sediment transport model with parameters which are easy to calibrate, related to previous well known theory, and with the potential to apply to random waves. We start from the BBB formula.

\section{THE BAGNOLD MODEL AND THE BBB CONCEPT}

Bagnold (1963, 1966) derived a stream-based sediment transport model assuming the sediment is transported in two modes: bedload and suspended load. Bedload sediment is transported by the flow via grain

\footnotetext{
${ }^{1}$ Center for Applied Coastal Research, University of Delaware, Newark, DE 19716, USA
} 
to grain interactions. Suspended sediment transport is supported by fluid flow through turbulent fluctuations. The total load sediment transport rate $i$ for 1-D flow is given as (Bagnold, 1966)

$$
i=\left[\frac{\epsilon_{b}}{\tan \phi-\tan \beta}+\frac{\epsilon_{s}\left(1-\epsilon_{b}\right)}{\left(w / u_{b}\right)-\tan \beta}\right] \Omega
$$

where $\Omega$ is energy dissipation inside the bottom boundary layer, $w$ is the fall velocity of sediment, $\epsilon_{b}$ and $\epsilon_{s}$ are the bedload and suspended load efficiencies, respectively, $\tan \beta$ is the bottom slope, and $\phi$ is the particle friction angle. The available fluid power, $\Omega$, is the work done by the bottom shear stress $\tau_{b}$, represented in two horizontal directions by

$$
\Omega=\tau_{b} \cdot \mathbf{u}_{b}
$$

where $\mathbf{u}_{b}$ is the nearbed free stream velocity. The bottom shear stress is parameterized using the quadratic drag law

$$
\boldsymbol{\tau}_{b}=\rho c_{f}\left|\mathbf{u}_{b}\right| \mathbf{u}_{b}
$$

with $c_{f}$ the bottom friction coefficient.

Substituting (2)-(3) into (1), and considering the bottom slope effect, Bailard (1981) obtained the time-averaged total sediment transport rate $<\mathbf{i}>$ written in terms of free stream velocity as

$$
\begin{aligned}
<\mathbf{i}> & =\rho c_{f} \frac{\epsilon_{b}}{\tan \phi}\left[<\left|\mathbf{u}_{b}\right|^{2} \mathbf{u}_{b}>-\frac{\tan \beta}{\tan \phi}<\left|\mathbf{u}_{b}\right|^{3}>\right] \\
& +\rho c_{f} \frac{\epsilon_{s}\left(1-\epsilon_{b}\right)}{w}\left[<\left|\mathbf{u}_{b}\right|^{3} \mathbf{u}_{b}>-\frac{\epsilon_{s}\left(1-\epsilon_{b}\right)}{w} \tan \beta<\left|\mathbf{u}_{b}\right|^{5}>\right],
\end{aligned}
$$

where $<>$ denotes time averaging over one wave period $T$. In (4), the nearbed free stream velocity, $\mathbf{u}_{b}$, is taken to be the near bed orbital velocity. The Bagnold model (1)-(3) and its wave-averaged version (4) relate the total sediment transport rate to the nearbed free stream velocity, implicitly neglecting any information on potential flow acceleration or pressure gradient effects within the bottom boundary layer. In particular, they predict zero sediment transport in wave fields with zero velocity skewness.

\section{A REFORMULATED BAGNOLD MODEL}

In this study, we instead take the approach of computing bottom shear stress and friction velocity $\mathbf{u}_{*}$ to examine whether a parameterization of the expression for work (2) in terms of boundary layer properties can lead to successful transport predictions. In the following, (3) is replaced with the expression

$$
\boldsymbol{\tau}_{b}=\rho\left|\mathbf{u}_{*}\right| \mathbf{u}_{*}
$$

which is the fundamental definition of friction velocity. Following Bagnold (1963), the available fluid power $\Omega$ is expressed in terms of the bottom shear stress and an unknown velocity $\mathbf{u}^{\prime}$ which remains to be estimated, giving

$$
\Omega=\tau_{b} \cdot \mathbf{u}^{\prime}
$$

A reasonable choice must be made for $\mathbf{u}^{\prime}$, which would be represented by free stream velocity $\mathbf{u}_{b}$ in the original Bagnold formulation. In order to avoid complicating the phase structure of $\Omega$, we use the shear velocity to determine the temporal behavior of $\mathbf{u}^{\prime}$, and write

$$
\mathbf{u}^{\prime}=c_{r} \mathbf{u}_{*}
$$

which gives the expression

$$
\Omega=\rho c_{r}\left|\mathbf{u}_{*}\right|^{3}
$$

for total work. Here $c_{r}$ is model parameter. Analysis of the magnitude of $c_{r}$ will be shown in detail in the journal version (Zhao et al 2010). Finally, substituting (8) into (1), the modified Bagnold formula is

$$
\mathbf{i}_{m}=\rho \frac{\epsilon_{b} c_{r}}{\tan \phi}\left[\left|\mathbf{u}_{*}\right|^{2} \mathbf{u}_{*}-\frac{\tan \beta}{\tan \phi}\left|\mathbf{u}_{*}\right|^{3}\right]+\rho \frac{\epsilon_{s}\left(1-\epsilon_{b}\right) c_{r}}{w}\left[\left|\mathbf{u}_{*}\right|^{3} \mathbf{u}_{*}-\frac{\epsilon_{s}\left(1-\epsilon_{b}\right)}{w} \tan \beta\left|\mathbf{u}_{*}\right|^{5}\right]
$$

where the subscript $m$ stands for modified sediment flux. After wave-averaging, the modified BBB model becomes

$$
\begin{aligned}
<\mathbf{i}_{m}>= & \rho \frac{\epsilon_{b} c_{r}}{\tan \phi}\left[<\left|\mathbf{u}_{*}\right|^{2} \mathbf{u}_{*}>-\frac{\tan \beta}{\tan \phi}<\left|\mathbf{u}_{*}\right|^{3}>\right] \\
& +\rho \frac{\epsilon_{s}\left(1-\epsilon_{b}\right) c_{r}}{w}\left[<\left|\mathbf{u}_{*}\right|^{3} \mathbf{u}_{*}>-\frac{\epsilon_{s}\left(1-\epsilon_{b}\right)}{w} \tan \beta<\left|\mathbf{u}_{*}\right|^{5}>\right]
\end{aligned}
$$




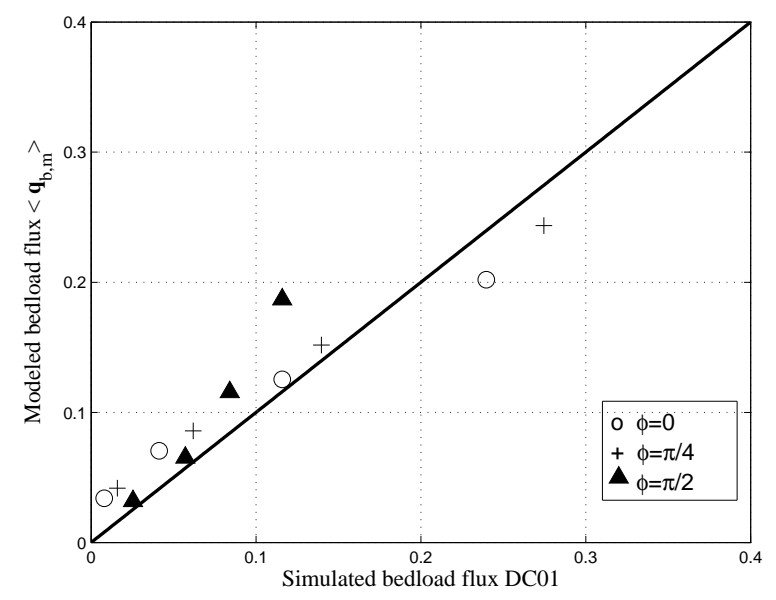

Figure 1. Simulated bedload flux from Drake and Calantoni (2001) versus modeled bedload flux , $<q_{b, m}>$, from Equation (11). Solid line represents a one-to-one comparison, $c_{r}$ is fixed to 8 and, $\epsilon_{b}$ is obtained from best fit of the data as 0.42 .

For cross-shore transport of coarse sediment on a horizontal bottom, the bedload flux $<\mathbf{q}_{b, m}>$ is

$$
<\mathbf{q}_{b, m}>=\frac{\rho_{s}}{g\left(\rho_{s}-\rho\right)} \rho c_{r} \frac{\epsilon_{b}}{\tan \phi}\left[<\left|\mathbf{u}_{*}\right|^{2} \mathbf{u}_{*}>\right.
$$

where $\rho$ and $\rho_{s}$ are fluid and sediment density, respectively. The corresponding bedload flux from BBB (4) is

$$
<\mathbf{q}_{b}>=\frac{\rho_{s}}{g\left(\rho_{s}-\rho\right)} \rho c_{f} \frac{\epsilon_{b}}{\tan \phi}\left[<\left|\mathbf{u}_{b}\right|^{2} \cdot \mathbf{u}_{b}>\right]
$$

\section{RESULTS}

The friction velocity $\mathbf{u}_{*}$ in (11) is calculated using a $k-\omega$ bottom boundary layer (BBL) model (Puleo $e t$ al., 2004). Figure (1) shows simulated bedload flux from Drake and Calantoni(2001) versus modeled bedload flux , $<q_{b, m}>$, from Equation (11), whereas (2) shows simulated net transport rate from Dohmen-Janssen and Hanes (2002) versus modeled net transport rate. Significant improvements in model skills can be found for these cases.

Next, to make the model more useful, it is worthwhile to explore the results using an analytical solution for bottom shear stress. Here we apply Madsen (1994) solution, in which the friction factor and phase shift are expressed using the relative roughness $r / A$, where $r$ is the Nikuradse roughness length, and $A=u_{b \max } T / 2 \pi$ is the wave orbital amplitude. Then friction velocity is calculated using the free stream velocity and phase shift following Nielsen (1992) and Nielsen and Callagnhan (2003). Once again, the model correlates well with data.

\section{SUMMARY}

We re-derived the Bagnold formula and showed that the net onshore directed sediment transport is correlated to the bottom shear stress. The original BBB-type formula written in terms of odd moments of free stream velocity was derived using the parameterized bottom shear stress for quasi-steady was unable to predict net onshore sediment transport under zero skewed sawtooth waves. The reformulated BBB model using a friction velocity obtained from a BBL model (Puleo et al. 2004) and a semi-analytical model (Madsen, 1994) is tested on Drake and Calantoni (2001) data as well as wave flume data of Dohmen-Janssen and Hanes (2002). Results collapse well with data, and show promising future.

\section{ACKNOWLEDGMENTS}

This research was funded by the National Oceanographic Partnership Program (N00014-99-1-1051), the Office of Naval Research, Coastal Geosciences Program (N00014-04-1-0219), and the University of Delaware. Comments from Qingping Zou are gratefully acknowledged. We also thank Dohmen-Janssen and Dan Hanes for sending us the data. 


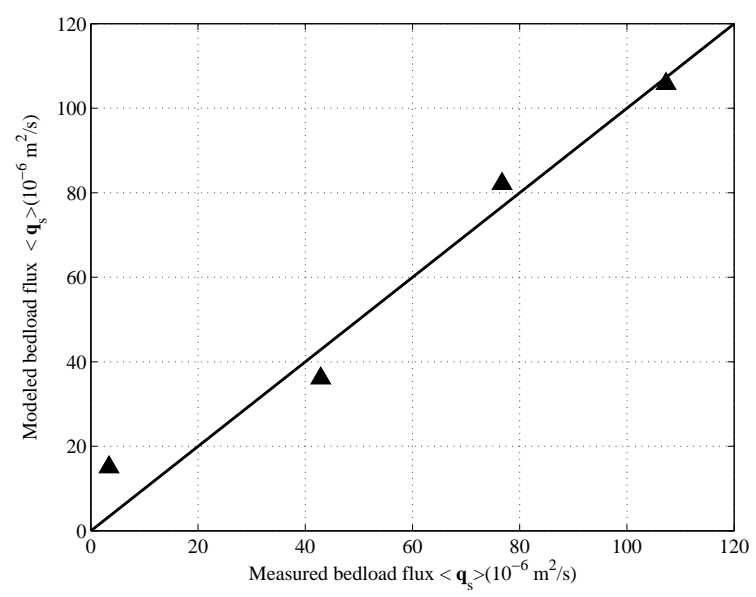

Figure 2. Measured net transport rate from Dohmen-Janssen and Hanes(2002) versus modeled net transport rate using Equation (11). Solid line represents a one-to-one comparison. $c_{r}$ is fixed to 8 and, $\epsilon_{b}$ is obtained from best fit of the data as 0.86 .

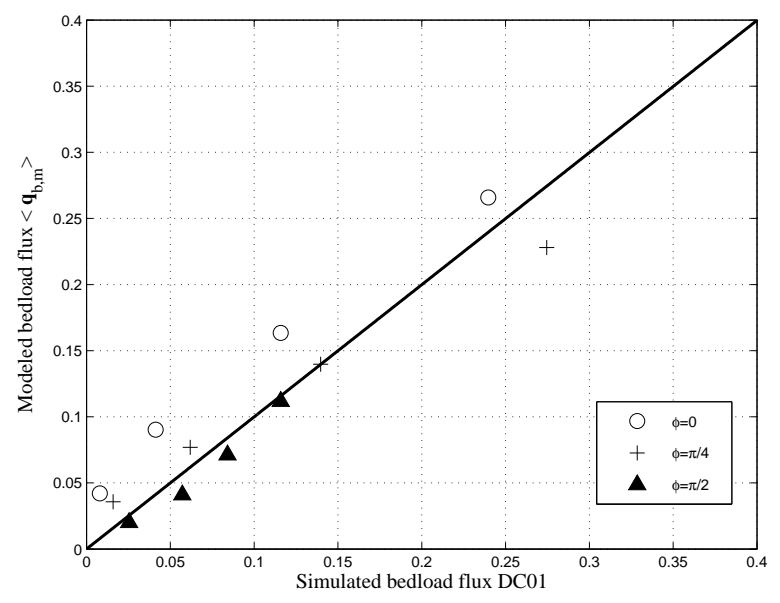

Figure 3. Simulated bedload flux from Drake and Calantoni(2001) versus modeled bedload flux,$<q_{b, m}>$, from Equation (11) using analytical solution of Madsen(1994). Solid line represents a one-to-one comparison. The best fit bedload efficiency for this calculation is $\epsilon_{b}=0.45$ and $c_{r}=8$.

\section{REFERENCES}

Bagnold, R. A. (1963) Mechanics of marine sedimentation, The Sea: Ideas and Observations, M. N. Hill ed, Interscience, Vol. 3, 507-528.

Bagnold, R. A. (1966) An approach to the sediment transport problem from general physics, Prof. Paper 422-1, U. S. Geol. Surv.

Bailard, J. A. (1981) An energetics total load sediment transport model for a plane slopping beach, J. Geophys. Res., 86 (C11), 10938-10954.

Bowen, A. J. (1980) Simple models of nearshore sedimentation: Beach profiles and longshore bars, In Coastline of Canada, 1-11, Halifax. Geological Survey of Canada.

Calantoni, J. and J. A. Puleo (2006) Role of pressure gradients in sheet flow of coarse sediments under sawtooth waves, J. Geophys. Res., 111, C01010, doi:10.1029/2005JC002875.

Dohmen-Janssen, C. M., and D. M. Hanes(200): Sheet flow dynamics under monochromatic nonbreaking waves, J. Geophys. Res., 107(C10), 3149, doi:10.1029/2001JC001045.

Drake, T. G. and J. Calantoni (2001) Discrete particle model for sheet flow sediment transport in the nearshore, J. Geophys. Res., 106 (C9), 19859-19868.

Elgar, S., E. L. Gallagher and R. T. Guza (2001) Nearshore sandbar migration, J. Geophys. Res., 106 (C6), 


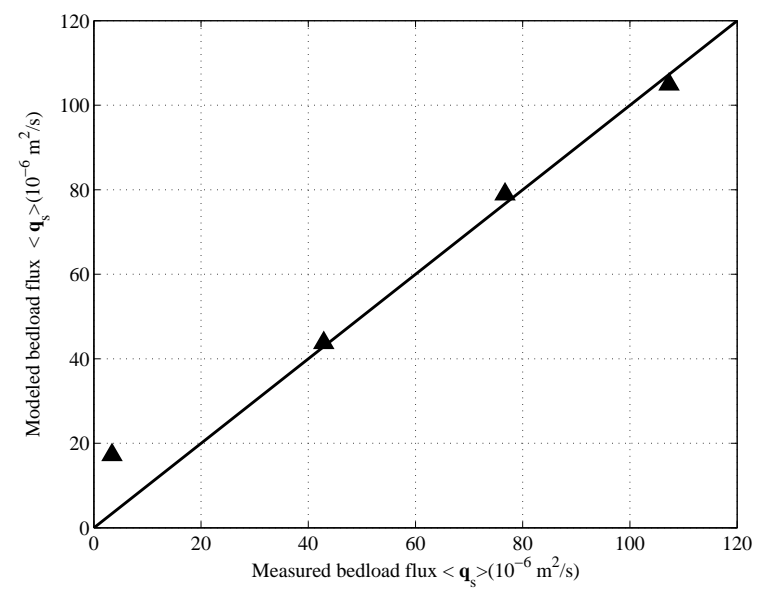

Figure 4. Measured net transport rate from Dohmen-Janssen and Hanes(2002) versus modeled net transport rate using analytical solution of Madsen(1994). Solid line represents a one-to-one comparison. The best fit bedload efficiency for this calculation is $\epsilon_{b}=0.73$. $c_{r}=8$.

\section{$11623-11627$.}

Foster, D. L., A. J. Bowen, R. A. Holman, and P. Natoo (2006), Field evidence of pressure gradient induced incipient motion, J. Geophys. Res., 111, C05004, doi:10.1029/2004JC002863.

Gallagher, E. L., S. Elgar and R. T. Guza (1998) Observation of sand bar evolution on a natural beach, J. Geophys. Res., 103 (C2), 3203-3215.

Gonzalez-Rodriguez, D. and Madsen, O. S. (2008): Bedload transport due to asymmetric and skewed waves plus a current. Proc. of 31st International Conference on Coastal Engineering (ICCE2008). pp 1596-1605.

Guard, P. A. and P. Nielsen (2008): unsteady flow effects on bed shear stress and sheet flow sediment transport. Proc. of 31st International Conference on Coastal Engineering (ICCE2008). pp 1521-1532.

Henderson, S., J. S. Allen and P. A. Newberger (2004) Nearshore sandbar migration predicted by an eddy-diffusive boundary layer model, J. Geophys. Res., 109, C06024, doi:10.1029/2003JC002137.

Hoefel, F. and S. Elgar (2003) Wave-induced sediment transport and sandbar migration, Science, 299, 1885-1887.

Hsu, T. -J. and D. M. Hanes (2004) The effect of wave shape on sheet flow sediment transport, J. Geophys. Res., 109, C05025.

Nielsen, P. (2002) Shear stress and sediment transport calculations for swash zone modelling. Coastal Engineering, 45, 53-60.

Nielsen, P. and D. P. Callaghan (2003) Shear stress and sediment transport for sheet flow under waves. Coastal Engineering, 47, 347-354.

Puleo, J. A., O. Mouraenko and D. M. Hanes (2004) One-dimensional wave bottom boundary layer model comparison: specific eddy viscosity and turbulence closure models, J. Waterway, Port, Coast. Ocean Engrng., 130 (6), 322-325.

Ruessink, B. G., T. J. J. van den Berg, and L. C. van Rijn (2009), Modeling sediment transport beneath skewed asymmetric waves above a plane bed, J. Geophys. Res., 114, C11021, doi:10.1029/2009JC005416.

Sleath, J. F. A. (1999) Conditions for plug formation in oscillatory flow, Continental Shelf Research, 19, 1643-1664.

Zhao, Q., J. T. Kirby and J. A. Puleo (2010): Bagnold formula revisited: Can energetics models be used to model on-shore sediment transport? In preparation. 